\title{
Reflets
}

Revue d'intervention sociale et communautaire

\section{Pratiques féministes, éducation populaire et conscience identitaire : le 5e colloque du RCFO}

\section{Jacinthe Michaud}

Volume 9, numéro 1, printemps 2003

Le genre en contexte : pratiques sociales et représentations

URI : https://id.erudit.org/iderudit/010866ar

DOI : https://doi.org/10.7202/010866ar

Aller au sommaire du numéro

Éditeur(s)

Reflets : Revue ontaroise d'intervention sociale et communautaire

ISSN

1203-4576 (imprimé)

1712-8498 (numérique)

Découvrir la revue

Citer cet article

Michaud, J. (2003). Pratiques féministes, éducation populaire et conscience identitaire : le 5e colloque du RCFO. Reflets, 9(1), 180-185.

https://doi.org/10.7202/010866ar

Tous droits réservés (C) Reflets : Revue ontaroise d'intervention sociale et communautaire, 2002
Ce document est protégé par la loi sur le droit d'auteur. L'utilisation des services d'Érudit (y compris la reproduction) est assujettie à sa politique d'utilisation que vous pouvez consulter en ligne.

https://apropos.erudit.org/fr/usagers/politique-dutilisation/ 


\title{
Pratiques féministes, éducation populaire et conscience identitaire
}

\author{
Jacinthe Michaud \\ École d'Études des femmes, Collège de Glendon
}

C'est dans le but d'analyser les effets des politiques néo-libérales sur l'éducation populaire, la santé et les services à l'intérieur des groupes de femmes que le Réseau des chercheures féministes francophones de l'Ontario a organisé sa $5^{\mathrm{e}}$ biennale autour du thème Pratiques féministes, éducation populaire et conscience identitaire. Ce colloque avait également pour objectif de mettre en lumière les nouveaux champs de communication et la reconfiguration des espaces publics/politiques afin de mieux rendre compte des manifestations de résistance et des nouvelles exigences de solidarité sociale.

Chercheures, intervenantes féministes, étudiantes francophones, toutes ont favorisé le succès de cette rencontre qui a eu lieu à Toronto, les 19-20-21 avril 2002, au Collège universitaire Glendon de l'UniversitéYork. Fidèle à sa tradition, le Réseau des chercheures féministes francophones de l'Ontario a recherché et a obtenu la participation de conférencières venues de l'extérieur de ses frontières. Ses nombreuses affiliations maintenues tant au Québec qu'à l'étranger, avec des regroupements similaires de chercheures féministes ont permis la convergence de communications portant sur de multiples enjeux de résistance et de pratiques novatrices. L'organisation thématique de ces trois jours de conférence a permis de regrouper les intérêts autour de cinq grands thèmes, ainsi que d'une table ronde. Cette dernière, organisée par la Table féministe francophone de concertation provinciale (TFFCP) s'est développée autour du sujet Recherche et communauté : un regard sur les réalisations de la TFFCP. Cette table ronde a donné le ton à 
l'ensemble des communications, cependant que Pierrette Bouchard, titulaire de la Chaire Claire-Bonenfant de l'Université Laval a prononcé l'allocution de clôture du colloque sur le thème "Les héritières du féminisme : perception et représentation de l'égalité dans vingt familles québécoises ".

\section{Les thèmes du Colloque}

La santé est constamment mise au coeur des préoccupations des groupes de femmes et de la recherche universitaire féministe et ce, dans diverses disciplines. Des communications sur cette question ont été présentées tout au long du colloque, et en particulier lors de la table ronde, Recherche et communauté : un regard sur les réalisations de la TFFCP en recherche-action, ainsi que lors de la séance suivante, Représentations sociales : ménopause et avortement. La Table féministe francophone de concertation provinciale (TFFCP) a réalisé plusieurs recherches-action ces dernières années, lesquelles ont fait l'objet d'une présentation de la part de Christine Dallaire et d'Angèle Bassolé. Par ailleurs, Lyne Bouchard, responsable de l'organisation de cette table, a présenté les principes qui gouvernent ce type de recherche féministe, ainsi que les principales activités que la Table féministe francophone entend poursuivre dans l'avenir. On en trouve un compte rendu dans ce numéro.

Dans Représentations sociales : ménopause et avortement, les participantes au colloque ont eu droit à deux communications, la première, de Monique Benoit, avait pour titre "Le recours à l'avortement et l'éducation sexuelle chez les jeunes femmes : une analyse comparative des 15-19 ans et des 20-24 ans ". Cette recherche visait à établir la relation entre le contenu des programmes d'éducation sexuelle dans les écoles secondaires et le recours à l'avortement chez deux groupes d'adolescentes en Ontario. La deuxième présentation «Ménopause et mitan de vie: deux phénomènes, une symbolique ", rendue par Christiane Bernier, constitue un des articles du " Dossier » du présent numéro. 
La session Questions de pouvoir : politique locale et globalisation, a reçu le plus grand nombre de communications; elle est, en outre, celle qui a le plus exploré les transformations politiques, économiques et sociales, aussi bien à l'échelon local avec les fusions municipales au Québec et en Ontario, qu'international avec la relocalisation des grandes industries vers les pays du Tiers-monde. Nicole Brais, du Centre de recherche en aménagement et développement de l'Université Laval, nous a fait part d'une expérience novatrice en éducation populaire avec "L'espace politique local et les groupes de femmes : l'émergence d'une nouvelle dynamique au Québec ». Les groupes de femmes de la ville de Québec n'ont pas attendu l'avènement de la fusion municipale pour se regrouper et revendiquer que le comité chargé de la transition vers la fusion et les élu-es municipaux s'engagent à préserver et à défendre les mécanismes de représentation des intérêts des femmes dans la nouvelle ville. L'auteure fait voir que durant toute la période de transition, le collectif «Femmes et restructuration municipale» a suscité de nombreuses occasions de débats et d'échange avec la population de la ville de Québec, créant ainsi des espaces d'éducation populaire sur certains enjeux féministes qui émergent avec plus d'acuité lors des périodes de transition.

La communication de Caroline Andrew et de Linda Cardinal, «La relève francophone en politique municipale : un enjeu pour les femmes " analysait une situation similaire de fusion municipale mais, cette fois, à Ottawa, ville bilingue et capitale nationale. On trouve un résumé du rapport produit sur la question, sous la rubrique Aux quatre coins de la province, dans le présent numéro.

Par ailleurs, Dominique Masson nous a déplacées vers une autre scène. Dans «Les avatars de la représentation : formation des discours politiques et représentation des femmes dans le développement régional ", elle s'intéresse aux dimensions discursives de la représentation des femmes dans l'espace régional, c'est-à- dire aux conditions de possibilités et aux conséquences d'une telle participation sur le discours politique, tant féministe qu'institutionnel. Pour terminer cette section, Jacinthe Michaud, dans "Les femmes, l'État et l'économie solidaire ", a fait état 
d'une recherche en cours en s'interrogeant sur la résurgence contemporaine du phénomène de l'économie sociale et de son foisonnement discursif, tant du côté académique que du côté communautaire, gouvernemental et même du secteur privé. À l'intérieur de ces ensembles discursifs, le secteur communautaire, selon l'auteure, constitue à la fois le terrain et l'objet des nouvelles orientations en politiques publiques dans la gestion des services de santé et des services sociaux. Elle se propose donc, dans sa recherche à venir, de faire l'analyse des divers corpus discursifs dans le champ de l'économie sociale et solidaire et de déterminer la part qu'y ont prise les théories féministes et anti-racistes dans l'ensemble de cette production.

Lu en format synthèse lors de cette même session, l'article de Nadia Naï, «Globalisation, délocalisation des entreprises et travail des femmes : l'exemple de l'entreprise hollandaise T.K. Fish dans la province de Tétouan dans le Nord du Maroc ", est aussi publié dans le présent numéro de Reflets.

Analyses de regroupements de femmes a donné lieu à des présentations fort diversifiées - mais intéressantes - quant aux débats qu'elles ont suscités et aux enjeux que leur contenu représente pour le mouvement des femmes. Avec «Le paradoxe du féminisme et du multiculturalisme canadien : un regard à partir des femmes africaines immigrantes ", Gertrude Mianda nous a fait part de sa recherche, qu'elle a assortie d'entrevues qualitatives auprès des femmes africaines et immigrantes des villes de Montréal et de Toronto. Selon Mianda, le vécu des femmes immigrantes africaines dévoile les contradictions de deux discours d'inclusion : féminisme et multiculturalisme. Il en étale les limites au point de confirmer les revendications féministes postcoloniales.

Pour sa part Denise Lemire dans "Coup d'oeil sur la pauvreté... rapport d'une étude portant sur les facteurs contribuant à la pauvreté des femmes francophones au Manitoba "nous a livré les premiers jalons d'une recherche entreprise pour le compte de Réseau femmes au Manitoba. Comme dernière communication, dans cette session, Renée St-Onge fait état d'une recherche portant sur le monde en transition des infirmières, lors d'une restructuration d'hôpitaux. Une version remaniée est 
reproduite dans ce numéro, sous la rubrique "Dossier", sous le titre «Fusion d'hôpitaux au Nord-Est de l'Ontario : quel espace pour la parole des infirmières?".

Dans Analyse de pratiques, de politiques et de services, nous avons eu droit à des présentations relatant les services spécifiques et les pratiques féministes dans certaines régions de l'Ontario, de même qu'à une analyse de discours de politique chez certaines catégories d'intervenant-es d'entreprises. Marie-Luce Garceau nous a fait un compte rendu de sa nouvelle recherche "Pour des services en français en violence dans le Nord ". Il s'agit d'une rechercheaction sur les besoins, les services et les modèles de prestation des services en français en matière de violence dans le Nord de l'Ontario. Cette recherche comporte deux volets : identification des besoins et des services et exploration des modèles de prestations de services.

Les trois communications suivantes sont publiées dans ce numéro. Il s'agit de la recherche de Cécile Coderre et de Joanne Hart, "Pratiques d'intervention féministe auprès des femmes survivantes d'agression à caractère sexuel: le contexte francoontarien "; de l'analyse de Natalie Dupont et de Christiane Bernier «Politiques contre le harcèlement sexuel. Comparaison et perception des agents et des plaignantes "; et, finalement, des enquêtes de Michèle Ollivier et d'Ann Denis, exposées, sommairement, sous l'intitulé "L'utilisation des technologies d'information et de communication chez les femmes francophones en situation minoritaire au Canada ".

Cela représente l'essentiel du contenu du dernier Colloque du Réseau des chercheures féministes francophones de l'Ontario. Le comité organisateur composé de Christiane Bernier (Laurentienne), Cécile Coderre (Ottawa),Jacinthe Michaud (York) tient à remercier toutes les participantes à cet événement. Elles tiennent également à remercier les généreux donateurs qui ont permis que ces trois jours de rencontre puissent se dérouler convenablement. Nous tenons à les nommer ici. Il s'agit de l'École d'études des femmes de l'UniversitéYork, du programme d'Études des femmes du Collège universitaire Glendon, du programme 
d'études graduées de l'École d'études des femmes de l'Université York, du Centre de recherche féministe à York, du Collège universitaire Glendon et du Secrétariat aux affaires internationales du Canada (SAIC). 